\title{
BMJ Open Using nudges to promote physical activity and to reduce sedentary behaviour in the workplace: a scoping review protocol
}

\author{
Sarah Forberger (D) , ${ }^{1}$ Frauke Wichmann, ${ }^{1}$ Chiara Nicoletta Nicoletta Comito (D) ${ }^{2}$
}

To cite: Forberger $\mathrm{S}$, Wichmann F, Comito CNN. Using nudges to promote physical activity and to reduce sedentary behaviour in the workplace: a scoping review protocol. BMJ Open 2020;10:e038205. doi:10.1136/ bmjopen-2020-038205

- Prepublication history and additional material for this paper is available online. To view these files, please visit the journal online (http://dx.doi.org/10. 1136/bmjopen-2020-038205).

Received 03 March 2020 Revised 07 0ctober 2020 Accepted 16 October 2020

Check for updates

(C) Author(s) (or their employer(s)) 2020. Re-use permitted under CC BY-NC. No commercial re-use. See rights and permissions. Published by BMJ.

${ }^{1}$ Prevention and Evaluation, Leibniz Institute for Prevention Research and EpidemiologyBIPS, Bremen, Germany

${ }^{2}$ Faculty of Sport and Exercise Sciences, University of Rome

'Foro Italico', Roma, Lazio, Italy

Correspondence to

Dr Sarah Forberger;

forberger@leibniz-bips.de

\section{ABSTRACT}

Introduction Physical inactivity and sedentary behaviour are associated with numerous health problems and increasing risks of premature morbidity and mortality. Workplace health promotion with a focus on increasing physical activity (PA) and reducing sedentary behaviour is of growing interest. The concept of choice architecture with the use of nudges is a promising approach to influence decision making regarding health behaviours. It can help to understand why people often fail to act in their best interest, to follow well-informed preferences or to achieve their set goals. Nudges, the way the choice is presented, can help to overcome these challenges by using the same habits, biases or boundaries to alter our decision-making in favour of the more preferred behaviour. Aims of the scoping review will be to analyse (a) to what extent the concept of choice architecture is used in workplace health promotion to promote PA and/or to reduce sedentary behaviour and (b) which instruments (nudges) are used to archive that.

Methods and analyses Medline, Psychlnfo, Web of Science and CINHAL will be searched from 2009 until June 2020. Applying a two-level screening process, title and abstracts will be screened according to a set of predetermined inclusion and exclusion criteria. Included articles will be screened a second time to determine the extent to which choice architecture has been used. Analyses for publication year, location, setting and target group will be provided. Interventions will be analysed presenting the instruments used, number of studies per instrument, combinations of instruments and alteration of the environment. Outcome measures and results will be reported as they occur.

Ethics and dissemination Due to the nature of the scoping review, ethical concerns are minimal. No patient data will be included. Results are published in peer-review journals.

\section{BACKGROUND}

One in four adults has an insufficient level of physical activity (PA). ${ }^{1}{ }^{2}$ Physical inactivity and prolonged sedentary behaviour (SB) are associated with numerous health problems and increased risk of premature morbidity, disability and mortality. ${ }^{134}$ Extensive evidence has been summarised on the benefits of
Strengths and limitations of this study

Following methodology of the Joanna Briggs Institute for scoping review and reporting in accordance with the Preferred Reporting Items for Systematic Reviews and Meta-Analyses flow chart.

- Screening done by two researchers independently.

- Interventions that do not state that they use choice architecture as theoretical approach are not included.

Embase is not included because of limited access.

routine PA for individual health status, with protective effects in various chronic diseases (eg, cardiovascular disease, stroke, hypertension, colon and breast cancer, type 2 diabetes). Even small changes in PA levels are of high relevance for risk reduction and better health. ${ }^{1}$ The WHO emphasises the promotion of PA as a key objective of disease prevention and health promotion in the coming decade. Workplace health programmes offer many adults an opportunity to increase PA and reduce $\mathrm{SB}$ during the workday. ${ }^{2}$ Active commuting to and from work, active breaks, sit-stand desks or stair use prompts offer ample opportunities to increase PA as part of daily activities and can therefore contribute to increased productivity and reduced injury. ${ }^{56}$ Through the workplace, it is possible to influence health behaviour of a large population group, directly affect the physical, mental, economic and social well-being of workers and indirectly the health of their families. ${ }^{5}$ For example, according to Eurostat, $73.1 \%$ of the population aged 20-64 are employed in the EU-27 in 2019; what makes the workplace an important setting for health promotion. ${ }^{7}$

Numerous reviews explore the workplace as setting for public health interventions to promote PA or reduce $\mathrm{SB}$; for example, role of workplace health programmes to enhance PA to increase productivity and reduce 
absenteeism, ${ }^{8-11}$ by targeting musculoskeletal disorders, lower back pain, ${ }^{12}{ }^{13}$ chronic diseases ${ }^{14}$ and by or by calculating the costs saved with a healthier workforce. ${ }^{6}$ The workplace plays a role in obesity prevention, either through weight management programmes or through multilevel interventions that combine healthy diet and PA. ${ }^{15-17}$ Programmes exist for certain groups such as university staff, ${ }^{18}$ nurses ${ }^{19}$ healthcare workers ${ }^{20}$ or male workers. ${ }^{21}$ Another focus is intervention design and characteristics. There are reviews analysing methods used, ${ }^{22} 23$ theoretical approaches used to develop the intervention, ${ }^{24}$ role of participants characteristics, ${ }^{25}$ recruitment rates ${ }^{2526}$ or perspectives of the employees regarding feasibility and acceptability. ${ }^{27}$ There is an extensive body of literature analysing the effectiveness of workplace health promotion interventions, either PA promotion or SB reduction interventions or of multilevel intervention combining nutrition, well-being and PA/SB interventions. ${ }^{172128-34}$

Some reviews analysed intervention characteristics. Rongen et al analysed intervention characteristics of workplace health promotion interventions, focusing on the influence of population, study and intervention characteristics and quality on the effectiveness of the interventions. However, they do not report on the use of choice architecture as theoretical approach. ${ }^{34}$ Jirathananuwat et al described and classified workplace interventions to promote PA in their systematic meta-review. ${ }^{35}$ They identified 48 interventions, 22 interventions focus on predisposing and 17 interventions on enabling employees to have more PA. Of the 22 predisposing factors, 6 were information delivery, 5 were self-motivation and 11 were programme training. The enabling approaches were instrument resources and health service facilities. The reinforcing approaches were incentives and social support. The remaining interventions focused on the environmental development and policy regulation. However, since they specify the instruments used within the intervention, they do not indicate whether the intervention was designed according to choice architecture approaches.

Malik et al reviewed workplace health promotion PA interventions to explore the type of interventions used. They found three intervention types: exercise, counselling or information intervention. ${ }^{36} \mathrm{Chu}$ et al investigated different intervention strategies-such as multicomponent and environmental strategies-to reduce sitting time at the workplace. ${ }^{37}$ With regard to an ageing workforce Poscia et al conducted a review on workplace health promotion programmes for older employees emphasising the need for future research on well-designed and cost-effective interventions to improve work-related outcomes. ${ }^{38}$ However, all three reviews do not report on the use of choice architecture.

Despite all the studies and promising results, people have difficulties in changing their behaviour, even if they want to change their lifestyle, have the awareness, intention and ability to do so. Behavioural insights can help to understand why people often do not act in their best interest, follow well-informed preferences or fail to achieve their set goals. Nudges can help to overcome these challenges by using the same habits, biases or boundaries to alter human decision-making in favour of the more preferred behaviour. ${ }^{39}{ }^{40}$ While behavioural insights is an approach to alter the architecture in which choices are made, nudges can generally be seen as the way choices are presented, ${ }^{41}$ as the deliberated 'effort to channel people into making decisions that are best for them' ${ }^{42}$ Following Hansen et al nudges can be defined as

a function of any attempt at influencing people's judgment, choice or behaviour in a predictable way (1) that is made possible because of cognitive boundaries, biases, routines and habits in individual and social decision-making posing barriers for people to perform rationally in their own declared self-interests and which (2) works by making use of those boundaries, biases, routines, and habits as integral parts of such attempts. ${ }^{43}$

This approach has been used in different areas such as energy use, ${ }^{44}{ }^{45}$ finance, ${ }^{46}{ }^{47}$ food choice, ${ }^{48-54}$ consumer ${ }^{55} 56$ or environmental protection. ${ }^{57} 58$ In the field of PA/SB, Nocon et al analysed the role of point of choice prompts to increase PA. ${ }^{59}$ Landais analysed studies on microenvironmental choice architecture interventions that promoted PA or discouraged SB in adults. ${ }^{60}$ Eighty-six studies targeted PA, predominantly stair use, whereas two studies targeted SB and one study targeted both behaviours. The intervention techniques identified were prompting $(n=53)$, message framing $(n=24)$, social comparison $(n=12)$, feedback $(n=8)$, default change $(\mathrm{n}=1)$ and anchoring $(\mathrm{n}=1)$. Although 19 workplacerelated were included in the review, no results specifically related to them were reported. Forberger $e t$ al analysed choice architecture interventions to promote PA within the general population ${ }^{40}$ and Szaszi et al provided an overview of the areas in which choice architecture was most commonly used. The most studied area was health, with most studies aimed to change eating or drinking behaviour, followed by studies attempted to alter sustainability behaviour, consumer choice, prosocial behaviour, finance, transport and education. ${ }^{61}$ The setting in which the studies were conducted was not analysed. At present, we are not aware of a systematic review or map that analysed interventions using choice architecture instruments to promote PA and/or to reduce of SB in the workplace.

Therefore, the paper aims to give an overview of choice architecture techniques used to increase PA and/or to reduce $\mathrm{SB}$ in the workplace setting to enrich research and practice on nudges as effective tools in setting-based health promotion.

This scoping review aims to:

1. Provide an overview of the application of the choice architecture approach in workplace health promotion (frequency of use). 
Table 1 List of search themes and terms used for the search strategy

\begin{tabular}{|c|c|c|c|}
\hline \multicolumn{2}{|c|}{ Search themes } & \multirow{2}{*}{$\begin{array}{l}\text { Search terms } \\
\text { physical activit*, standing, walk*, active, exercise, active break*, sitting break }{ }^{*} \text {, } \\
\text { walking break }^{*} \text {, active transport }{ }^{*}, \text { commut }^{*} \text {, biking, cycling }\end{array}$} & \multirow{2}{*}{$\begin{array}{l}\text { Search type } \\
\text { Title/abstract }\end{array}$} \\
\hline A & Physical activity & & \\
\hline \multirow[t]{2}{*}{ B } & $\begin{array}{l}\text { Sedentary } \\
\text { behaviour }\end{array}$ & $\begin{array}{l}\text { sedentary behaviour }{ }^{*} \text {, sedentary behavior*, sedentary lifestyl }{ }^{\star} \text {, sedentary, sit, } \\
\text { sitting, seat, seated, inactive, physical inactivity }\end{array}$ & Title/abstract \\
\hline & & sedentary behavior & MeSH term \\
\hline C & & workplace, work, workforce & MeSH term \\
\hline \multirow[t]{2}{*}{$\mathrm{D}$} & Workplace health & health promotion & Title/abstract \\
\hline & & health promotion, occupational health service & MeSH term \\
\hline
\end{tabular}

2. Inform about the intervention characteristics (target group, nudge used, length, content, organisational context).

3. Give an overview on the mode of delivery (eg, analogue, digital, face-to-face).

4. Identify outcome measures.

\section{METHODS}

The methodology for this scoping review will follow the guidelines of the Joanna Briggs Institute. ${ }^{62}$ Although the scoping review differs from systematic reviews in that it does not focus on evidence-based synthesis, features of the systematic search process will be followed

As a scoping review, the underlying objectives are (a) to analyse the body of literature found for workplace health promotion programmes in regard to choice architecture, (b) clarify intervention characteristics and mode of delivery and (c) to identify outcome measures. ${ }^{63}$ The literature will be analysed and gaps identified for further research. ${ }^{63}$ The Preferred Reporting Items for Systematic Reviews and Meta-Analyses flow diagram, which is used for reporting in systematic reviews, will be used for a transparent report of the article flow. ${ }^{64} 65$

\section{Search strategy}

Search string

The research question will reflect the PICO elements. PICO is an acronym for population, intervention, comparator and outcome.

The study population covers the working population as we analyse workplace health promotion interventions related to the choice architecture literature to increase $\mathrm{PA}$ and to reduce SB within the working day. Studies mention the use of choice architecture techniques and nudges against other intervention techniques as a comparator. Primary outcome is increased of PA and/or reduced of SB. As the distribution of the choice architecture approach is a focus of the search, no predefined outcomes beyond effective/not effective will be used. All further outcomes mentioned will be collected and presented as an overview.
Identification of relevant keywords for the search string will be conducted by the team of authors with the help of a science librarian (table 1). Terms will be linked by using BOOLEAN logic. The search will be developed in an iterative process. ${ }^{66}$ The search string will be pretested in the PubMed database online supplemental file 1 and refined by examining the research results. The agreed search string will be adapted to other databases according to necessary changes.

\section{Languages}

No language restrictions are applied in the search. Due to the requirements of the literature databases for the indexing of journals, English titles and abstracts are also available for journals that publish in the national language. Thus, the language restriction can be omitted. We will exclude papers that are not published in English/ German during the full-text screening and mark them accordingly in order to record the number of papers to be excluded due to language reasons.

\section{Databases}

The search will be performed in the following bibliographic databases (table 2):

While the use of choice architecture approaches is not new and many studies in psychology ${ }^{67}$ could retrospectively be categorised as using nudges according to

\begin{tabular}{llll}
\hline \multicolumn{3}{l}{ Table 2} & \multicolumn{3}{l}{ Overview of bibliographic databases used } \\
\hline \multicolumn{2}{l}{ Database } & Provider & Time span \\
\hline 1 & Medline & PubMed & $2009-06 / 2020$ \\
2 & Psyclnfo & Ovid & $2009-06 / 2020$ \\
3 & $\begin{array}{l}\text { Science Citation } \\
\text { Index Expanded }\end{array}$ & $\begin{array}{l}\text { Web of } \\
\text { Science }\end{array}$ & $2009-06 / 2020$ \\
& $\begin{array}{l}\text { Social Science } \\
\text { Citation Index }\end{array}$ & & $2009-06 / 2020$ \\
& $\begin{array}{l}\text { Arts and Humanities } \\
\text { Citation Index }\end{array}$ & & $2009-06 / 2020$ \\
4 & CINAHL & EBSCO & $2009-06 / 2020$ \\
\hline
\end{tabular}


Table 3 Predefined data extraction sheet with categories of the extracted data and corresponding characteristics

\begin{tabular}{lll}
\hline Category & Characteristics \\
\hline 1 & Author & All authors of the publication \\
2 & Year & Year of publication \\
3 & Publication type & Journal article, report \\
4 & Origin & The country in which the intervention was carried out \\
5 & Study design & Study design used \\
6 & Workplace characteristics & Workplace setting of intervention specified organisational context: (1) industry, (2) size,
\end{tabular}
(3) area (urban or rural)

7 Aim of the intervention Aim of the intervention and targeted behaviour of the intervention (PA and/or SB with specific subdomain of activity behaviour, for example, standing, walking, stair use, active breaks, active transport)

8 Intervention Short description of the intervention: (1) content, (2) length (follow-up period)

9 Target group specifications Details reported about the target group such as: (1) age, (2) gender, (3) organisational level (eg, manager, subordinates, apprentices, trainees, other staff), (4) occupation type (eg, white-collar or blue-collar worker), (4) excluded persons (eg, disabled persons)

10 Implementation management

Responsibility for implementation (if specified): (1) decision maker, (2) executer of intervention (internal, eg, HR Management or external, eg, contractor, research team), (3) others involved

11 Mode of delivery Specified mode for intervention delivery: (1) analogue/conventional (eg, paper-pencil, poster), (2) digital (eg, apps, portals, wearable, email), (3) face-to-face (eg, meetings, through coach)

12 Choice architecture elements used

\section{Following the MINDSPACE approach ${ }^{70}$}

Messenger

Incentives

Norms

Default

Salience

Priming

Affect

Commitments

Ego
We are heavily influenced by who communicates information

Our responses to incentives are shaped by predictable mental shortcuts such as strongly avoiding losses

We are strongly influenced by what others do

We 'go with the flow' of preset options

Our attention is drawn to what is novel and seems relevant to us

Our acts are often influenced by subconscious cues

Our emotional associations can powerfully shape our actions

We seek to be consistent with our public promises, and reciprocate acts

We act in ways that make us feel better about ourselves

Typology of intervention in accordance with TIPPME ${ }^{71}$ : (1) placement: Availability, position, (2) properties: Functionality, presentation, size, information Any information on active design guidelines used

13 Choice architecture elements with special focus on microenvironment

14 Typology of used instruments with Any instrument mentioned special focus on technique use

15 Outcome measures Outcome variable(s) reported: PA-related and SB-related (eg, expenditure, step count, time spent sitting or standing) measurement instrument used: subjective and/or objective (eg, self-report, diary, wearable)

16 Intervention results Study results

PA, physical activity; SB, sedentary behaviour; TIPPME, typology of intervention in proximal physical microenvironments. 
Thaler and Sunstein, ${ }^{68}$ the conscious application of its underlying processes and knowledge on behavioural change and decision making gained new interest in research after 2009 following the publication of their ground-breaking book. ${ }^{68}$ Therefore, the starting point for the search will be in 2009. In addition, the reference lists of included articles will be screened for additional articles.

\section{Study inclusion criteria and screening procedure Inclusion and exclusion criteria}

Articles will be eligible for inclusion if they (a) cover interventions in the workplace, (b) aim at increasing PA or reducing $\mathrm{SB}$, (c) that are designed by using choice architecture and (d) and name the theoretical approach accordingly. Our aim is to determine the frequency of use. Therefore, no indirect classification is used. The explicit naming of the choice architecture approach is necessary to be eligible. (e) All study designs are applicable. While the search and the title/abstract screening will be done without language restrictions, during the full-text screening articles will be excluded not written in English/German. In this way the proportion of literature in other languages can be determined. Further, interventions aimed at non-communicable diseases, weight management, obesity, pain reduction or other disorders will be excluded.

\section{Screening strategy}

The screening process will be managed with the help of the reference management software EndNote and the online software Covidence. The first step will be to remove all duplicates in EndNote, first within each database and then across databases. A second de-duplication will be performed in Covidence. After all duplicates have been removed, Covidence will be used for the screening. The screening process will follow predefined inclusion and exclusion criteria and will be a two-step procedure.

To answer the scoping review question about the frequency of use, all titles and abstracts will be screened to determine, if the intervention is conducted in the workplace and aims at increasing PA and/or reducing SB. During the full-text screening, the methodological approach will be assessed to determine whether a choice architecture approach is used. Only papers stating that they used choice architecture will be included. The entire screening process is carried out by two independent reviewers. ${ }^{69}$ Any disagreements will be resolved either by consensus or by discussion with a third reviewer. If no decision can be reached, studies will be discussed with an expert not involved in the screening process.

If there are doubts about eligibility, studies will be included in the next step. If, after the screening process, the eligibility of the studies remains unclear, further information will be obtained, also by contacting the study authors. ${ }^{69}$

\section{Data extraction and coding strategy}

A number of relevant classification systems have evolved in the last years. For this study, the MINDSPACE framework ${ }^{70}$ and TIPPME as the "typology of intervention in proximal physical microenvironments' (eg, staircase ${ }^{71}$ will be used. A combination of both classification systems will be used because while MINDSPACE can be seen as a list of instruments that can be used to alter behaviour, TIPPME focuses on the microenvironment. MINDSPACE is the acronym for messenger, incentives, norms, defaults, salience, priming, affect, commitments, and ego. That are nine of the most robust (non-coercive) influences on behaviour and can be used as checklist when making policy. ${ }^{70}$

Information will be organised by predefined extraction categories with corresponding characteristics in an extraction sheet. The extraction sheet will be pretested on three randomly chosen studies and adapted in an iterative process beforehand. The predefined data extraction sheet is presented in table 3. If information is not reported ' $\mathrm{n} / \mathrm{a}$ ' will be stated. Data extraction will be done by one reviewer. A second reviewer will analyse $10 \%$ of the records for quality reasons.

\section{Research synthesis}

Only studies with full data extraction-hence those using behavioural interventions-will be specified in the results section. Research synthesis will be based on the items from the extraction sheet following a narrative synthesis which gives an overview of the proportion of interventions that use a choice architecture approach. Analyses for publication year, location, setting and target group (included and excluded persons) will be provided. In this process, intervention instruments will be analysed related to choice architecture concept, number of studies per instrument used and combinations of instruments. Furthermore, a special focus will be given to alterations in a specific environment and the use of digital approaches. Outcome measures (variables and instruments) and results will be reported as they occur. Additionally, tables will be used to present the results of the literature analysis.

\section{ETHICS AND DISSEMINATION}

Due to the nature of the scoping review, ethical concerns are minimal. No patient data will be included. The scoping review will collect, synthesise and present previously collected and published data. If ethical concerns arise, the respective data will be excluded. Findings will be published in a pertinent journal article and the authors will disseminate the results within their research network through stakeholders and presentations.

Acknowledgements The authors thank our research librarian for her very helpful and valuable support.

Contributors SF and CNNC contributed to the planning of the study. All authors drafted and revised the protocol. CNNC will conduct the database research. CNNC, SF and FW will conduct the screening, analysis of data and drafting of the manuscript. 
Funding The authors have not declared a specific grant for this research from any funding agency in the public, commercial or not-for-profit sectors.

Competing interests None declared.

Patient consent for publication Not required

Provenance and peer review Not commissioned; externally peer reviewed.

Supplemental material This content has been supplied by the author(s). It has not been vetted by BMJ Publishing Group Limited (BMJ) and may not have been peer-reviewed. Any opinions or recommendations discussed are solely those of the author(s) and are not endorsed by BMJ. BMJ disclaims all liability and responsibility arising from any reliance placed on the content. Where the content includes any translated material, BMJ does not warrant the accuracy and reliability of the translations (including but not limited to local regulations, clinical guidelines, terminology, drug names and drug dosages), and is not responsible for any error and/or omissions arising from translation and adaptation or otherwise.

Open access This is an open access article distributed in accordance with the Creative Commons Attribution Non Commercial (CC BY-NC 4.0) license, which permits others to distribute, remix, adapt, build upon this work non-commercially, and license their derivative works on different terms, provided the original work is properly cited, appropriate credit is given, any changes made indicated, and the use is non-commercial. See: http://creativecommons.org/licenses/by-nc/4.0/.

\section{ORCID iDs}

Sarah Forberger http://orcid.org/0000-0002-7169-675X

Chiara Nicoletta Nicoletta Comito http://orcid.org/0000-0003-0487-6539

\section{REFERENCES}

1 Rhodes RE, Janssen I, Bredin SSD, et al. Physical activity: health impact, prevalence, correlates and interventions. Psychol Health 2017;32:942-75

2 WHO. Global action plan on physical activity 2018-2030: more active people for a healthier world. Geneva: World Health Organization, 2018.

3 Proper KI, Singh AS, van Mechelen W, et al. Sedentary behaviors and health outcomes among adults: a systematic review of prospective studies. Am J Prev Med 2011;40:174-82.

4 Beaglehole R, Bonita R, Horton R, et al. Priority actions for the noncommunicable disease crisis. The Lancet 2011;377:1438-47.

5 Quintiliani L, Sattelmair J, Sorensen G. The workplace as a setting for interventions to improve diet and promote physical activity. Geneva: World Health Organization, 2008: 1-36.

6 van Dongen JM, Proper KI, van Wier MF, et al. Systematic review on the financial return of worksite health promotion programmes aimed at improving nutrition and/or increasing physical activity. Obes Rev 2011;12:1031-49.

7 Eurostat. Employment - annual statistics 2020. Available: https:// ec.europa.eu/eurostat/statistics-explained/index.php?title= Employment - annual statistics; [Accessed 31 Jul 2020].

8 Pereira MJ, Coombes BK, Comans TA, et al. The impact of onsite workplace health-enhancing physical activity interventions on worker productivity: a systematic review. Occup Environ Med 2015;72:401-12.

9 Pronk NP, Kottke TE. Physical activity promotion as a strategic corporate priority to improve worker health and business performance. Prev Med 2009;49:316-21.

10 Kerner I, Rakovac M, Lazinica B. Leisure-Time physical activity and absenteeism. Arh Hig Rada Toksikol 2017;68:159-70.

11 Cancelliere C, Cassidy JD, Ammendolia C, et al. Are workplace health promotion programs effective at improving presenteeism in workers? A systematic review and best evidence synthesis of the literature. BMC Public Health 2011;11:395.

12 Moreira-Silva I, Teixeira PM, Santos R, et al. The effects of workplace physical activity programs on musculoskeletal pain: a systematic review and meta-analysis. Workplace Health Saf 2016;64:210-22.

13 Asada F, Takano K. Physical therapy for musculoskeletal disorders of workers: role of physical therapists in occupational health. Nihon Eiseigaku Zasshi 2016;71:111-8.

14 Meng L, Wolff MB, Mattick KA, et al. Strategies for worksite health interventions to employees with elevated risk of chronic diseases. Saf Health Work 2017;8:117-29.

15 Weerasekara YK, Roberts SB, Kahn MA, et al. Effectiveness of workplace weight management interventions: a systematic review. Curr Obes Rep 2016;5:298-306.
16 Ni Mhurchu C, Aston LM, Jebb SA. Effects of worksite health promotion interventions on employee diets: a systematic review. BMC Public Health 2010;10:62.

17 Maes L, Van Cauwenberghe E, Van Lippevelde W, et al. Effectiveness of workplace interventions in Europe promoting healthy eating: a systematic review. Eur J Public Health 2012;22:677-83.

18 Plotnikoff R, Collins CE, Williams R, et al. Effectiveness of interventions targeting health behaviors in university and College staff: a systematic review. Am J Health Promot 2015;29:e169-87.

19 Torquati L, Pavey T, Kolbe-Alexander T, et al. Promoting diet and physical activity in nurses. Am J Health Promot 2017;31:19-27.

20 Brand SL, Thompson Coon J, Fleming LE, et al. Whole-system approaches to improving the health and wellbeing of healthcare workers: a systematic review. PLoS One 2017;12:e0188418.

21 Wong JYL, Gilson ND, van Uffelen JGZ, et al. The effects of workplace physical activity interventions in men: a systematic review. Am J Mens Health 2012;6:303-13.

22 Johnson S, Regnaux J-P, Marck A, et al. Understanding how outcomes are measured in workplace physical activity interventions: a scoping review. BMC Public Health 2018;18:1064.

23 Wierenga D, Engbers LH, Van Empelen P, et al. What is actually measured in process evaluations for worksite health promotion programs: a systematic review. BMC Public Health 2013;13:1190.

24 Hutchinson AD, Wilson C. Improving nutrition and physical activity in the workplace: a meta-analysis of intervention studies. Health Promot Int 2012;27:238-49.

25 Waters LA, Galichet B, Owen N, et al. Who participates in physical activity intervention trials? J Phys Act Health 2011;8:85-103.

26 Ryde GC, Gilson ND, Burton NW, et al. Recruitment rates in workplace physical activity interventions: characteristics for success. Am J Health Promot 2013;27:e101-12.

27 Baxter S, Sanderson K, Venn AJ, et al. The relationship between return on investment and quality of study methodology in workplace health promotion programs. Am J Health Promot 2014;28:347-63.

28 Malińska M. Effectiveness of physical activity intervention at workplace. Med Pr 2017;68:277-301.

29 To QG, Chen TTL, Magnussen CG, et al. Workplace physical activity interventions: a systematic review. Am J Health Promot 2013:27:e113-23.

30 Freak-Poli RLA, Cumpston M, Peeters A, et al. Workplace pedometer interventions for increasing physical activity. Cochrane Database Syst Rev 2013;4:Cd009209.

31 van Wier MF, van Dongen JMH, van Tulder MW. Worksite physical activity and nutrition programmes: beneficial to our health and wallet?. Ned Tijdschr Geneeskd 2013;157:A4963.

32 Conn VS, Hafdahl AR, Cooper PS, et al. Meta-Analysis of workplace physical activity interventions. Am J Prev Med 2009;37:330-9.

33 Barr-Anderson DJ, AuYoung M, Whitt-Glover MC, et al. Integration of short bouts of physical activity into organizational routine a systematic review of the literature. Am J Prev Med 2011;40:76-93.

34 Rongen A, Robroek SJW, van Lenthe FJ, et al. Workplace health promotion: a meta-analysis of effectiveness. Am J Prev Med 2013;44:406-15

35 Jirathananuwat A, Pongpirul K. Promoting physical activity in the workplace: a systematic meta-review. J Occup Health 2017;59:385-93.

36 Malik SH, Blake H, Suggs LS. A systematic review of workplace health promotion interventions for increasing physical activity. $\mathrm{Br} \mathrm{J}$ Health Psychol 2014;19:149-80.

37 Chu AHY, Ng SHX, Tan CS, et al. A systematic review and metaanalysis of workplace intervention strategies to reduce sedentary time in white-collar workers. Obes Rev 2016;17:467-81.

38 Poscia A, Moscato U, La Milia DI, et al. Workplace health promotion for older workers: a systematic literature review. BMC Health Serv Res 2016;16 Suppl 5:329.

39 Hansen PG, Skov LR, Skov KL. Making healthy choices easier: regulation versus Nudging. Annu Rev Public Health 2016;37:237-51.

40 Forberger S, Reisch L, Kampfmann T, et al. Nudging to move: a scoping review of the use of choice architecture interventions to promote physical activity in the general population. Int J Behav Nutr Phys Act 2019;16:77.

41 Hallsworth M, Snijders V, Burd H, et al. Applying behavioral insights: simple ways to improve health outcomes. Doha, Qata: World Innovation Summit for Health, 2016.

42 Schlag P. Nudge, choice architecture, and Libertarian paternalism. Michigan Law Review 2010;108:913-24.

43 Hansen PG. The definition of Nudge and Libertarian paternalism: does the hand fit the glove? European Journal of Risk Regulation 2016;7:155-74

44 Kaiser M, Bernauer M, Sunstein CR, et al. The power of green defaults: the impact of regional variation of opt-out tariffs on 
green energy demand in Germany. Ecological Economics 2020;174:106685.

45 Rasul I, Hollywood D. Behavior change and energy use: is a 'nudge' enough? Carbon Manag 2012;3:349-51.

46 Cai CW. Nudging the financial market? A review of the nudge theory. Account Finance 2019;2.

47 Cribb J, Emmerson C. What happens when employers are obliged to nudge? Automatic enrolment and pension saving in the UK. IFS Working Papers, 2016.

48 Bauer JM, Reisch LA. Behavioural Insights and (Un)healthy Dietary Choices: a Review of Current Evidence. J Consum Policy 2019;42:3-45.

49 Bucher T, Collins C, Rollo ME, et al. Nudging consumers towards healthier choices: a systematic review of positional influences on food choice. Br J Nutr 2016;115:2252-63.

50 Vandenbroele J, Vermeir I, Geuens M, et al. Nudging to get our food choices on a sustainable track. Proc Nutr Soc 2020;79:133-46.

51 Cheung TTL, Gillebaart M, Kroese FM, et al. Cueing healthier alternatives for take-away: a field experiment on the effects of (disclosing) three nudges on food choices. BMC Public Health 2019;19:974.

52 Broers VJV, De Breucker C, Van den Broucke S, et al. A systematic review and meta-analysis of the effectiveness of nudging to increase fruit and vegetable choice. Eur $J$ Public Health 2017;27:912-20.

53 Kraak VI, Englund T, Misyak S, et al. A novel marketing mix and choice architecture framework to nudge restaurant customers toward healthy food environments to reduce obesity in the United States. Obes Rev 2017:18:852-68.

54 Nørnberg TR, Houlby L, Skov LR, et al. Choice architecture interventions for increased vegetable intake and behaviour change in a school setting: a systematic review. Perspect Public Health 2016;136:132-42.

55 Sibony A-L, Helleringer G. EU consumer protection and behavioural sciences. In: Nudge and the Law : A European Perspective, 2015.

56 Reisch LA, Zhao MIN. Behavioural economics, consumer behaviour and consumer policy: state of the art. Behav Public Policy 2017;1:190-206.

57 Sunstein CR, Reisch LA. Automatically green: behavioral economics and environmental protection. Harv Envtl L Rev 2014;38:127.
58 Reisch L, Eberle U, Lorek S. Sustainable food consumption: an overview of contemporary issues and policies. Sustainability: Science, Practice and Policy 2013;9:7-25.

59 Nocon M, Müller-Riemenschneider F, Nitzschke K, et al. Review Article: Increasing physical activity with point-of-choice prompts--a systematic review. Scand J Public Health 2010;38:633-8.

60 Landais LL, Damman OC, Schoonmade LJ, et al. Choice architecture interventions to change physical activity and sedentary behavior: a systematic review of effects on intention, behavior and health outcomes during and after intervention. Int J Behav Nutr Phys Act 2020;17:47

61 Szaszi B, Palinkas A, Palfi B, et al. A systematic scoping review of the choice architecture movement: toward understanding when and why nudges work. J Behav Decis Mak 2018;31:355-66.

$62 \mathrm{JBI}$. Methodology for JBI Scoping Reviews. Joanna Briggs Institute Reviewers' Manual: 2015 Edition/Supplement. South Australia, Australia: The Joanna Briggs Institute, 2015.

63 Peters MDJ, Godfrey CM, Khalil H, et al. Guidance for conducting systematic scoping reviews. Int J Evid Based Healthc 2015;13:141-6.

64 Shamseer L, Moher D, Clarke M, et al. Preferred reporting items for systematic review and meta-analysis protocols (PRISMA-P) 2015: elaboration and explanation. BMJ 2015;349:97647.

65 Tricco AC, Lillie E, Zarin W, et al. PRISMA extension for scoping reviews (PRISMA-ScR): checklist and explanation. Ann Intern Med 2018;169:467-73.

66 Levac D, Colquhoun H, O'Brien KK. Scoping studies: advancing the methodology. Implement Sci 2010;5:69.

67 Marchiori DR, Adriaanse MA, De Ridder DTD. Unresolved questions in nudging research: putting the psychology back in nudging. Soc Personal Psychol Compass 2017;11:e12297.

68 Thaler RH, Sunstein CR. Nudge: improving decisions about health, wealth, and happiness. Penguin, 2009.

69 Reisch LA, Andor MA, Doebbe F, et al. Mitigating climate change in food consumption and food waste: a systematic map of behavioural interventions, search protocol for a systematic mapping study. Copenhagen/Stockholm/Essen: OSF, 2019.

70 Dolan P, Hallsworth M, Halpern D, et al. Mindspace: influencing behaviour for public policy, 2010.

71 Hollands GJ, Bignardi G, Johnston M, et al. The TIPPME intervention typology for changing environments to change behaviour. Nat Hum Behav 2017;1:1-9. 